\title{
Effect of mazEF, higBA and relBE toxin-antitoxin systems on antibiotic resistance in Pseudomonas aeruginosa and Staphylococcus isolates
}

\author{
Umut Safiye Say Coskun ${ }^{1}$, Aysegul Copur Cicek², Cetin Kilinc ${ }^{3}$, Ridvan \\ Guckan $^{3}$, Yelda Dagcioglu ${ }^{4}$, Osman Demir ${ }^{5}$, Cemal Sandallı \\ 1. Gaziosmanpasa University Faculty of Medicine, Department of Medical Microbiology, Turkey. \\ 2. Recep Tayyip Erdoğan University Faculty of Medicine, Department of Medical Microbiology, Turkey. \\ 3. Amasya University Sabuncuoglu Serafeddin Training and Research Hospital, Department of Microbiology, \\ Turkey. \\ 4. Gaziosmanpasa Universirty Training And Research Hospital, Microbiology Laboratory, Turkey. \\ 5. Gaziosmanpasa University School of Medicine, Department of Biostatistics, Turkey.
}

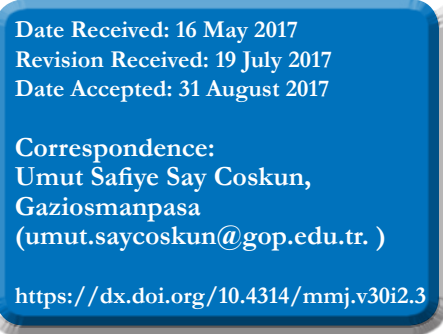

\section{Background}

\section{Abstract}

A toxin-antitoxin (TA) system is a set of two or more closely linked genes that are encoded as a poison and a corresponding antidote on a protein. In typical bacterial physiology, an antitoxin binds to a toxin and neutralizes it, which prevents the bacterium from killing itself. We aimed to determine whether P.aeruginosa and Staphylococcus isolates have TA genes and to investigate whether there is a relationship between the expression levels of TA genes and resistance to antibiotics.

Methods

This study included 92 P. aeruginosa and 148 Staphylococcus isolates. RelBE, higBA genes were investigated in P.aeruginosa by multiplex polymerase chain reaction (PCR). The mazEF gene and the all TA genes expression were detected by real time PCR.

Results

RelBE and higBA genes were detected in $100 \%$ of $P$. aeruginosa. It was found that the level of relBE TA gene expression is increased in isolates sensitive to aztreonam compared to resistant isolates $(\mathrm{p}<0.05)$. The mazEF gene was detected in $89.1 \%$ of Staphylococcus isolates. In terms of MazEF gene expression level there was no significant difference between methicillin-sensitive Staphylococcus aureus (MSSA) and methicillin-resistant $S$. aureus (MRSA) isolates ( $\mathrm{p}>0.05)$ whereas there was a significant difference between MSSA and coagulase-negative Staphylococcus (CNS) isolates, MRSA and CNS isolates $(\mathrm{p}<0.05)$. The levels of mazEF gene expression were found to be higher in isolates sensitive to gentamicin, ciprofloxacin, levofloxacin, clindamycin, phosphomycine, nitrofurantoin, fusidic acid, cefoxitin compared to resistant isolates $(\mathrm{p}<0.05)$.

Conclusion

Studies on the prevalence and functionality of TA systems emphasize that it may be possible to have new sensitive regions in bacteria by activating TA systems. The results of this study lead to the idea that resistance to antibiotics can be reduced by increasing TA gene expression levels. But there is need for further studies to support and develop this issue.

\section{Introduction}

Bacterial infections are increasingly prevalent due to rapid changes in the patient population and increased number of chronic diseases and immunosuppressed patients. The increase in antibiotic resistance complicates the treatment of Pseudomonas aeruginosa ( $P$. aeruginosa), coagulase-negative Staphylococcus (CNS) and especially $S$. aureus infections ${ }^{1}$.

$S$. aureus is a pathogen that can cause invasive infections such as endocarditis, osteomyelitis and sepsis as well as skin and soft tissue infections that can be colonized in humans and animals ${ }^{2}$. Although the pathogenicity of CNS is lower than that of $S$. aureus, it has been observed to be more frequently isolated in invasive infections in recent years. In the United States, 80.461 invasive methicillin-resistant S. aureus (MRSA) infections were reported in 2011, of which 11.285 resulted in death. In the same study, it was found that approximately 51.000 hospital-acquired P.aeruginosa infections occurred, of which $13 \%$ were due to multi drug resistance (MDR) $P$. aeruginosa and 400 resulted in death. Along with a reduction in the number of antibiotics that can be used in the treatment of infections, infections that cannot be controlled indicate a universal danger ${ }^{3}$.
A toxin-antitoxin (TA) system is a set of two or more closely linked genes that are encoded as a poison and a corresponding antidote on a protein. In typical bacterial physiology, an antitoxin binds to a toxin and neutralizes it, which prevents the bacterium from killing itself. When the antitoxin is degraded or not functional, the toxin kills the bacterium; this is known as a programmed cell death ${ }^{4}$.

TA systems are genes encoded on chromosomes and plasmids $^{5}$ that can be found in both Gram-negative and Gram-positive bacteria ${ }^{6}$. Studies over the past 30 years have revealed detailed information about the functions and movement mechanisms of TA systems as well as various interesting results regarding the importance of such systems for bacterial physiology ${ }^{7,8}$.

Generally, toxin molecules act as negative regulators for cell life, whereas antitoxin molecules act as positive regulators. The interaction between toxin and antitoxin gene expression levels in stressful conditions is vital for the life of the bacteria. Therefore, studies are being conducted on the possibility that TA systems can be used to develop new antibiotics ${ }^{9-11}$.

Bacteria often have more than one TA system in their genome $^{12}$. The presence and type of TA systems and whether 

the then bacteria? To our knowledge, there is no study showing the existence $\mathrm{pM}$ of each primer, $10 \mu \mathrm{L}$ reaction buffer (Promega), $3 \mu \mathrm{L}$ of TA systems in methicillin-sensitive Staphylococcus aureus $25 \mathrm{mM} \mathrm{MgCl} 2,200 \mu \mathrm{M}$ of each dNTP and $1.5 \mathrm{U}$ of Taq (MSSA) and CNS isolates. The aim of this study was Polymerase (Promega, USA). PCR amplification conditions to determine whether P.aeruginosa isolates isolated from were as follows: initial denaturation at $94^{\circ} \mathrm{C}$ for 3 minutes clinical specimens have relBE Table 1: Primers used for detection of TA genes and analysis of real time PCR and higBA TA systems and whether Staphylococcus isolates in P. aeruginosa and Staphylococus clinical isolates.

have mazEF TA systems, and

to investigate whether there Primer name 5'-3' Sequence Expected Fragment, bp Application Reference is a relationship between the mazEF

and resistance to antibiotics.

\section{Methods}

\section{Ethical Information}

This study was approved
by the Scientific and

Ethical Committee of the

Clinical Research Enthics

Committee (Tokat, Turkey),

(16-KAEK-061/03.03.2016).

F-

$408 \quad 18$

R-
AGAAGGATATTCACAAATGCTGA

F-

505

R-ATGAGCACCGTAGTCTCGTTC

F- CTCATGTTCGATCTGCTTGC
Malawi Medical Journal 30 (2): 67-72 June 2018

Total RNA isolation from $P$. aeruginosa and Staphylococcus isolates

The prepared $30 \pm 5 \mathrm{ng} / \mu \mathrm{L}$ bacterial suspension was RB buffer (RB buffer, $\beta$ - Mercaptoethanol) was added to the pellet and RNA isolation was performed according to the pellet and $\mathrm{N}$. Reaction mix, $7.5 \mu$ of DNase I and $1725 \mu \mathrm{L}$ of $10 \mathrm{x}$ Rece added for degradation of the genomic DNA dasing the protocol and pure RNA was obtained.

Table 2: Distribution of clinical specimens of $P$. aeruginosa and Staphylococcus isolates.

\begin{tabular}{|l|l|l|}
\hline Microorganism & $P$. aeruginosa & $\begin{array}{l}\text { Staphylococcus } \\
\text { isolates }\end{array}$ \\
\hline Sample type & $\mathrm{n} / \%$ & $\mathrm{n} / \%$ \\
\hline $\begin{array}{l}\text { Endotracheal } \\
\text { aspirate }\end{array}$ & $34 / 37$ & $4 / 8.3$ \\
\hline Blood & $23 / 25$ & $87 / 58.8$ \\
\hline Wound & $10 / 10.8$ & $24 / 16.2$ \\
\hline $\begin{array}{l}\text { Bronchoalveolar } \\
\text { lavage }\end{array}$ & $9 / 9.7$ & - \\
\hline Pleural effusion & $5 / 5.4$ & - \\
\hline Catheter & $4 / 4.3$ & $1 / 0.7$ \\
\hline Urine & $3 / 3.3$ & $6 / 4$ \\
\hline Abscess & $3 / 3,3$ & $3 / 2$ \\
\hline Tissue & $1 / 1.1$ & - \\
\hline Joint fluid & - & $1 / 0.7$ \\
\hline Conjunctiva & - & $1 / 0.7$ \\
\hline $\begin{array}{l}\text { Cere bros pin al } \\
\text { fluid }\end{array}$ & - & $6 / 4$ \\
\hline Sputum & - & $15 / 10.1$ \\
\hline
\end{tabular}

Preparation of cDNA from total RNA in $P$ aeruginosa and Staphylococcus isolates:

The cDNA was prepared by adding $10 \mu \mathrm{L}$ of water, $8 \mu \mathrm{L}$ of reaction mix and $2 \mu \mathrm{L}$ of reverse transcriptase (RT), to a final volume of $20 \mu \mathrm{L}$. The cDNA was prepared for a total $22^{\circ} \mathrm{C}, 30$ minutes at $42^{\circ} \mathrm{C}, 5$ minutes at $85^{\circ} \mathrm{C}$. The identity of the cell number of the resulting cDNAs was confirmed by measuring with NanoDrop spectrophotometer. The activity of the gene region was proven by the detection of the CDNI using SYBR green dye.

Detection of TA gene expression in P. aeruginosa and Staphylococcus isolates by real time PCR

The mazEF and relBE genes were prepared by adding 12.5 $\mu \mathrm{L}$ of Super SYBR Mix and for the relBE and higBA genes, $0.5 \mu \mathrm{L}(+)$ and $(-)$ of primer and for the mazEF gene, 0.25 $\mu \mathrm{L}(+)$ and $(-)$ of primer, $6.5 \mu \mathrm{L}$ of water, and $3 \mu \mathrm{L}$ of
cDNA, to obtain a total of $20 \mu \mathrm{L}$ mix. The amplification cDNA, to obtain a total of $20 \mu \mathrm{L}$ mix. The amplification 45 cycles of 15 seconds denaturation at $95^{\circ} \mathrm{C}$ for the RelBE 45 cycles of 15 seconds denaturation at $95^{\circ} \mathrm{C}$ for the RelBE
primer binding at $56^{\circ} \mathrm{C}$ for 45 seconds, for the HigBA
Toxin-antitoxin systems on antibiotic resistance 69 mer binding at $52^{\circ} \mathrm{C}$ for 45 seconds, and for the MazEF 作然er binding at $54^{\circ} \mathrm{C}$ for 30 seconds and then elongation at $72^{\circ} \mathrm{C}$ for 30 seconds, followed by a final elongation step by

able 3: Antibiotic resistance rates of $P$. aeruginosa and Staphylococcus isolates.

\begin{tabular}{|l|l|l|l|}
\hline \multicolumn{2}{|l|}{ P. aeruginosa } & \multicolumn{2}{l|}{ Staphylococcus isolates } \\
\hline Antibiotic & $n$ n $\%$ & Antibiotic & $n$ n $\%$ \\
\hline Meropenem & $59 / 67$ & Penicillin & $126 / 95.5$ \\
\hline Imipenem & $52 / 59.8$ & Sefoksitin & $84 / 63.6$ \\
\hline $\begin{array}{l}\text { Cefoperazone- } \\
\text { sulbactam }\end{array}$ & $36 / 42.9$ & Gentamicin & $36 / 27.3$ \\
\hline Ceftazidime & $20 / 23.8$ & Erythromycin & $101 / 7.6$ \\
\hline $\begin{array}{l}\text { Piperacillin- } \\
\text { tazobactam }\end{array}$ & $19 / 21.8$ & Clindamycin & $41 / 31$ \\
\hline Ciprofloxacin & $19 / 21.8$ & Linezolid & 0 \\
\hline Cefepime & $14 / 15.9$ & Daptomycin & 0 \\
\hline Aztreonam & $12 / 13.6$ & Teicoplanin & 0 \\
\hline & & Vancomycin & 0 \\
\hline & & Ciprofloxacin & $44 / 33.3$ \\
\hline & & Levofloxacin & $31 / 23.5$ \\
\hline & & Tetracycline & $45 / 34$ \\
\hline & & Fusidic acid & $35 / 26.5$ \\
\hline & & $\begin{array}{l}\text { Trime th o p rim- } \\
\text { sulfamethoxazole }\end{array}$ & $22 / 16.6$ \\
\hline
\end{tabular}

\section{Statistical Method}

In study, Shapiro-Wilk's test was used to assess the data normality. When the data of the Staphylococcus isolates were the normally distributed independent variables between two rroups and Mann-Whitney U test was used to compare the non normally distributed independent variables between two croups. One-way ANOVA test was used in thee groups comparisons. For multiple comparisons, the Tukey HSD test was used. The difference between resistance to antibiotics in $P$. eeruginosa isolates and transcription levels of TA genes was investigated with an independent samples t test. The statistical significance level of $\mathrm{p}$ was 0.05 . Statistical analysis was performed using commercial software (IBM SPSS Statistics 20, SPSS Inc., an IBM Co., Somers, NY).

Results

P. aeruginosa and isolates were identified in respiratory tract specimens (36 endotracheal aspirates and 9 bronchoalveola lavages) at a rate of $46.7 \%$, whereas Staphylococcus isolates were most frequently identified in blood culture samples at a rate of $58.8 \%$. The sample types in which the isolates were identified are shown in Table 2 . In the $P$. aeruginosa isolates, aztreonam was identified as the most sensitive antibiotic type, whereas the highest resistance rates were for $\frac{\text { for meropenem and imipenem, respectively. The antibiotics }}{\text { https://dx.doi.org/10.4314/mmj.v30i2.3 }}$ 
Table 4: Antibiotic resistance status and mazEF cT values of $\begin{aligned} & \text { most resistant against Staphylococcus isolates were penicillin } \\ & (95.5 \%) \text { and erythromycin }(76.5 \%) \text {. Resistance rates against }\end{aligned}$ Staphylococcus isolates

\begin{tabular}{|c|c|c|c|c|}
\hline \multicolumn{2}{|l|}{ Factors } & \multicolumn{2}{|c|}{ MazEF Ct Values } & \multirow{2}{*}{$\mathrm{p}$} \\
\hline \multicolumn{2}{|l|}{$\mathrm{n}$} & Mean $\pm S D$ & & \\
\hline \multirow{3}{*}{ Group } & MSSA & 48 & $23.3 \pm 5.42$ & \multirow{3}{*}{$<0.001$} \\
\hline & MRSA & 51 & $21.37 \pm 5.85$ & \\
\hline & CNS & 33 & $32.04 \pm 4.63$ & \\
\hline \multirow[t]{2}{*}{ Penicillin } & Sensitive & 6 & $25.9 \pm 6.24$ & \multirow{2}{*}{$0.751^{*}$} \\
\hline & Resistant & 126 & $24.68 \pm 6.94$ & \\
\hline \multirow{2}{*}{ Gentamicin } & Sensitive & 96 & $23.98 \pm 6.44$ & \multirow{2}{*}{0.038} \\
\hline & Resistant & 36 & $26.76 \pm 7.7$ & \\
\hline \multirow{2}{*}{ Ciprofloxacin } & Sensitive & 88 & $23.51 \pm 6.14$ & \multirow{2}{*}{0.007} \\
\hline & Resistant & 44 & $27.19 \pm 7.68$ & \\
\hline \multirow{2}{*}{ Levofloxacin } & Sensitive & 101 & $23.82 \pm 6.35$ & \multirow{2}{*}{0.015} \\
\hline & Resistant & 31 & $27.71 \pm 7.81$ & \\
\hline \multirow[t]{2}{*}{ Erythromycin } & Sensitive & 31 & $22.81 \pm 6.88$ & \multirow{2}{*}{0.074} \\
\hline & Resistant & 101 & $25.33 \pm 6.82$ & \\
\hline \multirow{2}{*}{ Clindamycin } & Sensitive & 91 & $23.75 \pm 6.69$ & \multirow{2}{*}{0.013} \\
\hline & Resistant & 41 & $26.93 \pm 6.9$ & \\
\hline \multirow{2}{*}{ Linezolid } & Sensitive & 132 & $24.67 \pm 6.87$ & \\
\hline & Resistant & 0 & & \\
\hline \multirow{2}{*}{ Daptomycin } & Sensitive & 132 & $24.74 \pm 6.89$ & \\
\hline & Resistant & 0 & - & \\
\hline \multirow{2}{*}{ Teicoplanin } & Sensitive & 132 & $24.73 \pm 6.92$ & \\
\hline & Resistant & 0 & & \\
\hline \multirow[t]{2}{*}{ Vancomycin } & Sensitive & 132 & $24.73 \pm 6.92$ & \\
\hline & Resistant & 0 & & \\
\hline \multirow[t]{2}{*}{ Tetracycline } & Sensitive & 87 & $24.63 \pm 7.12$ & \multirow{2}{*}{0.807} \\
\hline & Resistant & 45 & $24.94 \pm 6.48$ & \\
\hline \multirow{2}{*}{ Fusidic acid } & Sensitive & 97 & $23.81 \pm 6.42$ & \multirow{2}{*}{0.010} \\
\hline & Resistant & 35 & $27.3 \pm 7.56$ & \\
\hline \multirow{2}{*}{$\begin{array}{l}\text { Trimethoprim- } \\
\text { sulfamethoxazole }\end{array}$} & Sensitive & 110 & $24.86 \pm 6.86$ & \multirow[b]{2}{*}{0.662} \\
\hline & Resistant & 22 & $24.15 \pm 7.15$ & \\
\hline \multirow{2}{*}{ Cefoxitin } & Sensitive & 48 & $23.3 \pm 5.42$ & \\
\hline & Resistant & 84 & $25.56 \pm 7.51$ & 0.048 \\
\hline Inducible & Sensitive & 87 & $25.57 \pm 6.15$ & \\
\hline $\begin{array}{l}\text { clinda my y in - } \\
\text { resistance }\end{array}$ & Resistant & 6 & $35.96 \pm 6.36$ & $0.002^{*}$ \\
\hline
\end{tabular}

MSSA: Methicillin-sensitive Staphylococcus aureus; MRSA: Methicillin-resistant S. Aureus; CNS: Coagulase negative staphylococcus, antibiotics are shown in Table 3. HigBA and relBE genes were detected in $100 \%$ of the isolates $(\mathrm{n}=92)$. For each screened gene, the sample was displayed by running on the agarose gel (Figure 1). The mazEF gene was detected in 132 (89.1\%) of 148 Staphylococcus isolates. The resistance rates of isolates to antibiotics and the average cycle threshold $(\mathrm{Ct})$ values for the mazEF gene are shown in Table 4. The mean Ct values were $23.9 \pm 8.8$ for the relBE gene and $18.24 \pm$ 5.8 for the higBA gene. The RelBE TA gene expression leve was found to be increased in isolates sensitive to aztreonam compared to resistant isolates $(\mathrm{p}<0.05)$. The distribution of MazEF values by groups is shown in Figure 2. In term of MazEF gene expression levels, there was no significan difference between MSSA and MRSA isolates $(\mathrm{p}=0.181$ ), and $\mathrm{CNS}$ isolates $(\mathrm{p}<0.001)$ and MRSA and CNS isolates $(p<0.05)$. The levels of mazEF gene expression were foun to be higher in isolates sensitive to gentamicin, ciprofloxacin, levofloxacin, clindamycin, nitrofurantoin, fusidic acid an cefoxitin compared to resistant isolates $(\mathrm{p}<0.05)$. The level of gene expression was found to be higher in isolates withou inducible clindamycin-resistance compared to thoswith inducible clindamycin resistance $(\mathrm{p}<0.05)$.

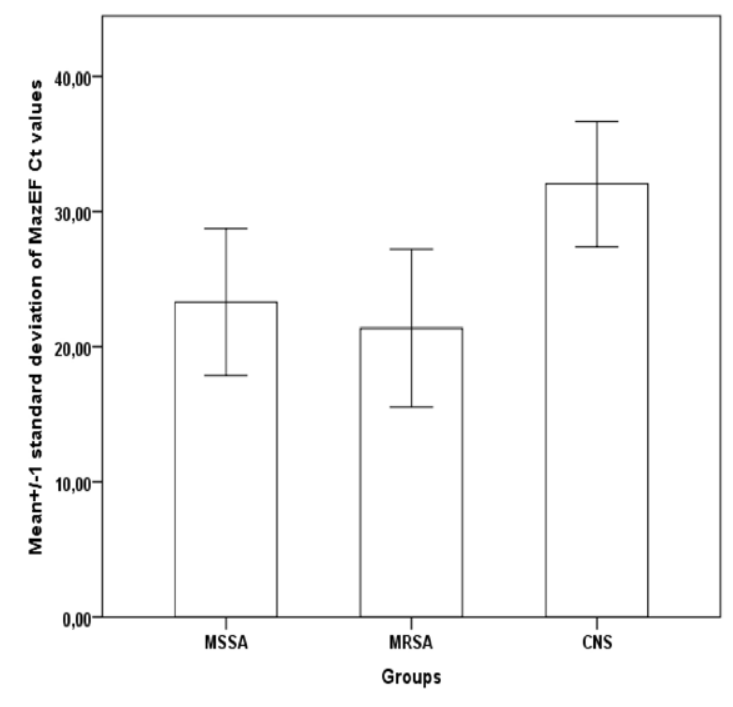

\section{Discussion}

Bacteria often have more than one TA system in their genome $^{12}$. The presence and type of TA systems and whether they are encoded on a plasmid or on a chromosome varies between bacteria ${ }^{4}$. TA systems are thought to be encoded on chromosomes in the P. aeruginosa $a^{1,1,6}$. Based on aeruginosa, including PAQ1, relBE, higBA, and parDE ${ }^{17,18}$ However, a study by Gang Li et al. in 2016 identified a new TA gene named hibAB in the P. aeruginosa ${ }^{19}$. The studies have also revealed the existence of new TA systems. The genes of seven known TA gene families for S. aureus have been identified, with the majority of the investigation involving the mazEF TA gene ${ }^{17,18}$. MazEF, an operon that is called a "plasmid addiction system" and which stabilize plasmids, was first identified on the chromosome of $E$ coli in 1993. MazEF is the most studied TA system in E. coli and whereas there was significant difference between the MSSA

\section{Figure 2: The distribution of MazEF values by groups in}

e mediator in cell death ciprofloxacin, levofloxacin, clindamycin, phosphomycin, in stress conditions ${ }^{20}$ as well as a modulator in the translation process $^{2,22}$

RelBE is also one of the most studied TA systems in $E$. coll $h^{2}$. It modulates the response induced in the case of amino acid starvation ${ }^{2}$, which leads to the inhibition of translation and consequently to bacteriosis ${ }^{21}$. In the literature, E. coli appears to be the most frequently investigated bacterium for $\mathrm{TA}$ systems. HigBA was first described on Protens vulgaris ${ }^{23}$. In a study conducted in the United States, it was reported that TA genes were encoded on the chromosome in P. aeruginosa and S. aureus isolated from clinical samples collected from three centers and that $100 \%$ of the 78 MRSA isolates had higBA and relBE, whereas $30 \%$ had parDE and all $42 P$. aeruginosa isolates had relBE and higBA TA genes. The authers of the study confirmed the PCR products by DNA sequencing, with the result that $97.8 \%$ to $100 \%$ of the PCR products were compatible with the DNA sequencing. In addition, they also emphasized that these genes are transcribed and that the activation of toxin genes would be an effective antibacterial strategy $^{15}$. In a study conducted in 2016, 174 P. aeruginosa isolates isolated from clinical specimens were found to have relBE, higBA and parDE TA genes at a rate of $100 \%, 100 \%$ and $30 \%$, respectively in Iran . Another study conducted in Iran by Hemati et al. showed that $P$. aeruginosa isolates had mazEF TA genes at a rate of $85.7 \%$ and that the isolates were resistant to gentamicin $(65 \%)$, meropenem $(60 \%)$ found a corelation betwe found a correlation between mazEF genes and resistance this study, higBA and relBE genes were found in all $(100 \%)$ of $P$. of $P$. aerminosa isolates, while the mazEF gene was found in (the literature and show tha are consistent whe the literature and show that TA genes are present at high rates in $P$. aerminosa and Staphylococcus gene expression were found to be higher in isolates sensitive to gentamicin, ciprofloxacin, levofloxacin, clindamycin, phosphomycin, nitrofurantoin, fusidic acid and cefoxitin isolates, in contrast to the study by Hemati et al ${ }^{24}$, the relBE TA gene expression level was found to be inceesed in isolates sensitive to aztreonam compared to resistant isod $(\mathrm{p}<0.05)$. For Staphylococcus isolates, the existence of TA systems has been proven only in MRSA isolates, while these systems remained unexplored in CNS and MSSA isolates. In this study we evaluated the presence of TA systems in MSSA and CNS and observed the transcription of the mazEF TA gene in MRSA and MSSA isolates is higher than in CNS $(p<0.05)$. This sugrests that the mazEF TA rene may be related to virulence in $S$, aureus. To our knowledge, this is the first study investigating TA genes in the CNS The determination of the prevalence of TA genes in strains with less virulence will also contribute to the studies investigating the possibility that TA systems are antibacterial targets. Bukowski et al. ${ }^{25}$ suggests that TA systems induce bacteriosis, which in the long run result in the death of bacterial cells. The results of Bukowski et al..$^{25}$ and Hemati et al..$^{24}$ also point out that drugs that can activate silent toxins in TA systems can be used as antibiotics. The present study is consistent with the results of Bukowski et al. ${ }^{25}$ and Hemati et $\mathrm{al}^{24}$ in terms of the finding that isolates with higher mazEF gene expression levels were more sensitive to gentamicin,
政, the present study, the toxin and antitoxin genes were evaluated together because they were located on the same operon. Bacteria were not exposed to any stress factors, such as heat, amino acid pestiction, or antibiotic exposure. We investigated only the pesence of these TA systems and the association between

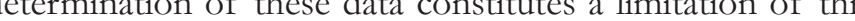
study. The studies on the prevalence and functionality of The possible to have new Thitive regions in bacteria by activating TA systems (by degrading antitoxins or increasing toxin expression), matter of fact the results of this study lead to the iden antiotics can be reduced by increasing $\mathrm{TA}$ is expression levels. The most important step at this stage to investigate the presence and functionality of TA genes in geses in bacteria to support and develop this strategy and to deternite for this target. Ou P. P.

\section{Conclusion}

Studies on the prevalence and functionality of TA system emphasize that it may be possible to have new sensitive his study lead to the idea that resistance to antibiotics can be educed by increasing TA gene expression levels. But tere is need for further studies to support and develop this issue.

This work was supported by Gaziosmanpasa University Research Fund Grants (BAP-2015/25).

\section{Conflict of interests}

All authors declare that they have no competing interests elated to this work.

\section{References}

. Becker K, Heilmann C, Coagulase-negative staphylococci, Peters G.

2. Franklin D, Lowy M.D. Staphylococcus aureus Infections. N Engl Med. 1998; 339: 520-532. DOI: 10.1056 NEJM199808203390806

3. Center for Diseases Control and Prevention (CDC). (2013). Antibiotic cesistance Threats in The United States. Atlanta,
cdcov/drugresistance/pdf/ar-threats-2013-508.pd

4. Gerdes K. Unique type of plasmid maintenance function: USA. 1986; 83: 3116-3120.

5. Gentry DR, Hernandez VJ, Nguyen LH, Jensen DB, Cashel M. Synthesis of the stationary-phase sigma factor $\sigma \mathrm{S}$ is positively regulated y ppGpp. J. Bacteriol 1993;175: 7982-7989.

6. Moritz EM, Hergenrother PJ. Toxin-antitoxin systems are ubiquitou 7. Kwan BW, Valenta JA, Benedik MJ, Wood TK. Arrested protein synthesis increases persister-like cell formation. Antimicrob Agen
Chemother 2013:57: 1468-1473. DOI- 10.1128/AAC.02135-12 heir presence and antibiotic resistance. Only the statistical 政

\section{Acknowledgements} lin Microbiol Rov. 2014, 27(4):870 -926. DOl. 10.1128/CMR.00109and plasmid-encoded in vancomycin-resistant Enterococci. Proc Na 
8. Wood TL, Wood TK. The HigB/HigA toxin/antitoxin system of Pseudomonas aeruginosa influences the virulence factors pyochelin, pyocyanin, and biofilm formation. Microbiologyopen. 2016; 5(3): 499511. doi: $10.1002 / \mathrm{mbo} 3.346$

9. Engelberg-Kulka H, Sat B, Reches M, Amitai S, Hazan R. Bacterial programmed cell death systems as targets for antibiotics. Trends Microbiol 2004; 12: 66-71. doi: 10.1016/j.tim.2003.12.008

10. Lioy VS, Rey O, Balsa D, Pellicer T, Alonso JC. A toxin-antitoxin module as a target for antimicrobial development. Plasmid 2010; 63: 31-39. doi: 10.1016/j.plasmid.2009.09.005.

11. Park SJ, Son WS, Lee BJ. Structural overview of toxin-antitoxin systems in infectious bacteria: a target for developing antimicrobial agents. Biochim Biophys Acta 2013; 1834(6): 1155-1167. doi: 10.1016/j.bbapap.2013.02.027

12. Brown BL, Grigoriu S, Kim Y, Arruda JM, Davenport A. et. al. Three dimensional structure of the MqsR: MqsA complex: a novel TA pair comprised of a toxin homologous to RelE and an antitoxin with unique properties. PLoS Pathog. 2009; 5(12): e1000706.doi: 10.1371/ journal.ppat.1000706.

13. Clinical and Laboratory Standards Institute. Performance standards for antimicrobial susceptibility testing. 22nd Informational Supplement, M100-S22, 2012. CLSI, Wayne, PA.

14. Ausubel FM, Brient R, Kingston RE, Moore DD, Seidman JG, Smith JA et al. Short protocols in molecular biology, 2nd edn. New York: John Willey and Sons, 1995.

15. Williams JJ, Halvorsen EM, Dwyer EM, DiFazio RM, Hergenrother PJ. Toxin-antitoxin (TA) systems are prevalent and transcribed in clinical isolates of Pseudomonas aeruginosa and methicillin-resistant Staphylococcus aureus. FEMS Microbiol Lett. 2011; 322(1): 41-50. doi: 10.1111/j.1574-6968.2011.02330.x

16. Savari M, Rostami S, Ekrami A, Bahador A. Characterization of Toxin-Antitoxin (TA) Systems in Pseudomonas aeruginosa Clinical Isolates in Iran. Jundishapur J Microbiol. 2016; 9(1): e26627. doi: $10.5812 / \mathrm{jjm} .26627$
17. Pandey DP, Gerdes K. Toxin-antitoxin loci are highly abundant in free-living but lost from host-associated prokaryotes. Nucleic Acids Res. 2005; 33(3): 966-976. dOI: 10.1093/nar/gki201

18. Gerdes K, Christensen SK, Lobner-Olesen A. Prokaryotic toxinantitoxin stress response loci. Nat Rev Microbiol. 2005; 3: 371-382. doi: 10.1038/nrmicro1147

19. Li G, Shen M, Lu S, Le S, Tan Y. et al. Identification and Characterization of the HicAB Toxin-Antitoxin System in the Opportunistic Pathogen Pseudomonas aeruginosa. Toxins (Basel). 2016; 8(4): 113. doi: 10.3390/toxins 8040113

20. Amitai G, Shemesh A, Sitbon E, Shklar M, Netanely D. et. al. Network analysis of protein structures identifies functional residues. J Mol Biol. 2004; 344(4): 1135-1146. doi: 10.1016/j.jmb.2004.10.055

21. Pedersen K, Christensen SK, Gerdes K. Rapid induction and reversal of a bacteriostatic condition by controlled expression of toxins and antitoxins. Mol Microbiol 2002; 45(2): 501-510. doi: 10.1046/j.13652958.2002.03027.x

22. Christensen SK, Mikkelsen M, Pedersen K, Gerdes K. RelE, a global inhibitor of translation, is activated during nutritional stres. Proc Natl Acad Sci U S A. 2001; 98(25): 14328-14333. doi: 10.1073/ pnas. 251327898

23. Tian QB, Hayashi T, Murata T, Terawaki Y. Gene product identification and promoter analysis of hig locus of plasmid Rts1. Biochem. Biophys. Res. Commun. 1996; 225: 679-684. doi: 10.1006/ bbrc.1996.1229

24. Hemati S, Azizi-Jalilian F, Pakzad I, Taherikalani M, Maleki A. et al. The correlation between the presence of quorum sensing, toxinantitoxin system genes and MIC values with ability of biofilm formation in clinical isolates of Pseudomonas aeruginosa. Iran J Microbiol. 2014; 6(3): 133-139.

25. Bukowski M, Rojowska A, Wladyka B. Prokaryotic toxin-antitoxin systems: the role in bacterial physiology and application in molecular biology. Acta Biochim Pol. 2011; 58(1): 1-9. 